\title{
Characteristic Mode Theory of Wavetraps for Antenna Decoupling
}

\author{
Mikko Heino, Clemens Icheln, Pasi Ylä-Oijala, Buon Kiong Lau, Katsuyuki Haneda
}

\begin{abstract}
This paper introduces a systematic design method for decoupling elements, which can significantly improve the isolation between two co-located antennas, e.g. between transmit and receive antennas of an in-band full-duplex system. The design method applies the theory of characteristic modes for controlling the phase and amplitude of the scattered fields of the decoupling element, in order to optimally cancel the original incident fields which couple to the receiving antenna. We describe concisely the effects that characteristic angle, modal near-field, and modal excitation of the decoupling element have on the antenna isolation. For validating the proposed method, a planar wavetrap is designed and the isolation improvement verified with full-wave simulations. When we use the proposed method to optimize a wavetrap that is placed between two co-located patch antennas, we obtain an improvement of the isolation between the antennas by $33 \mathrm{~dB}$ at the centre frequency of their operational frequency band, and at least 12-dB improvement across the whole 142-MHz operational bandwidth of the two antennas. As a benchmark, the wavetrap is replaced by an absorber occupying 10 times the volume of the wavetrap. The absorber gives only $6 \mathrm{~dB}$ of isolation improvement, substantiating the effectiveness of the proposed wavetrap method.
\end{abstract}

Index Terms-Theory of characteristic modes, monopole antennas, patch antennas, mutual coupling, wavetrap, antenna isolation, scattering.

\section{INTRODUCTION}

High spectral efficiency requirement of modern communication systems increases the need for high antenna isolation in many applications. In multiple-input-multiple-output (MIMO) systems, high antenna isolation is needed to provide diversity gain and minimize the envelope correlation coefficient between antennas. In in-band full-duplex systems, very high antenna isolation is needed to provide enough isolation to make the transmitter invisible to the receiver and thus in theory double the spectral efficiency of the system.

One effective way to achieve decoupling is to introduce parasitic elements to the highly coupled antennas. So far, parasitic decoupling structures have mostly been designed by optimizing a known structure with parametric sweeps [1]-[4]. In [1], two closely placed antennas have been isolated by placing a decoupling element between them. Slots and split ring resonators have been used in the ground plane of the antennas for improved isolation [2]. In [3], the admittance

The research work leading to these results was funded by Aalto ELEC Doctoral School, Nokia Foundation and the HPY Research Foundation.

M. Heino, C. Icheln, P. Ylä-Oijala and K. Haneda are with the Department of Electronics and Nanoengineering, School of Electrical Engineering, Aalto University, FI-00076 Espoo, Finland (e-mail: firstname.lastname@aalto.fi)

B. K. Lau is with the Department of Electrical and Information Technology (EIT), Lund University, 22100 Lund, Sweden (e-mail: buon_kiong.lau@eit.lth.se). between antennas is cancelled by using coupled resonators between the antennas. A more rigorous approach uses a parasitic antenna for decoupling [5]. Circuit equations were derived to obtain the required load at the parasitic antenna to achieve perfect decoupling. It is shown that the required load can be made purely reactive by tuning the antenna elements. A typical method to describe the mutual coupling has been to derive a circuit model for the antenna system and then creating an additional path of coupling which neutralizes the original mutual admittance between the antennas [6], [7].

The theory of characteristic modes, first introduced by Garbacz and Turpin [8], is based on diagonalizing the scattering matrix of a conducting body to obtain orthogonal characteristic modes. The theory was then refined by Harrington and Mautz [9] using the linear operator theory to diagonalize the impedance matrix to obtain the same characteristic modes.

Characteristic modes are eigencurrents obtained by solving a weighted eigenvalue equation based on an integral equation formulation of a conducting structure [9]. These modes can be used to expand the induced surface current due to any excitation or incident field. Characteristic modes only depend on the shape and size of the conducting object, which enables antenna design in a physically intuitive and controlled way.

Characteristic modes have been used to design MIMO antennas with isolated modes for user devices with a small ground plane [10]-[12]. Antenna decoupling has been studied with characteristic modes by calculating the modal admittance between antennas and using reactive loading to suppress the unwanted modes [13], [14]. In [15], the eigenvalues of mutually coupled modes were noted to reduce when a parasitic resonator was added. However, these methods rely on calculating the modal admittance and selecting suitable loads for the arbitrarily placed parasitic elements. Hence, they are not easily applied when designing the shape of passive structures for increasing antenna isolation without lumped elements.

To the authors' knowledge, so far there exists no method to analytically design decoupling elements based on controlling the scattering of characteristic modes and resulting in cancellation of the original fields. In [16], the idea of using the phase of characteristic modes to engineer the scattering of elements and design directors for antennas was suggested. In this paper, it is shown that by carefully tuning the characteristic angles of different characteristic modes, the phase and amplitude of the scattered field can be tuned, so that the scattered field cancels the original incident field at the receiving antenna location. This approach enables physically intuitive decoupling element design using the characteristic mode theory.

The major problem we address in this paper is that no 
general model exists for antenna decoupling resonators that is based on the concept of scattered fields from the theory of characteristic modes. The main novelties of this paper are as follows:

1) Characteristic modes are applied to study the phase of scattered fields for antenna isolation.

2) A methodology for designing parasitic decoupling resonators and identifying all parameters affecting isolation is formulated.

The paper provides a solution on how to design the scattered fields of a wavetrap so that they cancel the original fields and thus create high isolation. With the characteristic mode theory, it is possible to separate the effects of the characteristic angle, incident wave exciting the resonator, and modal near-field on the isolation.

\section{WAVETRAP SCATTERING THEORY}

\section{A. Characteristic Angle}

In discrete form, the characteristic mode theory utilizes the modal decomposition of the impedance matrix obtained with method of moments (MoM) solution for analyzing electromagnetic problems. The eigenvalue decomposition for an arbitraryshaped perfect electric conductor (PEC) object in the theory of characteristic modes is formulated as

$$
X \mathbf{J}_{n}=\lambda_{n} R \mathbf{J}_{n},
$$

in which $Z=R+j X$ is the impedance matrix, and $\mathbf{J}_{n}$ the eigenvector or the characteristic current, and $\lambda_{n}$ the corresponding eigenvalue [9]. The total current induced on a conducting body can be expressed with the formula

$$
\mathbf{J}=\sum_{n} \alpha_{n} \mathbf{J}_{n}, \alpha_{n}=\frac{V_{n}}{1+j \lambda_{n}}, V_{n}=\oiiint_{S} \mathbf{J}_{n} \cdot \mathbf{E}_{i n c} d S,
$$

where $\alpha_{n}$ is the modal weighting coefficient, $\mathbf{E}_{i n c}$ is the incident field on the structure, $S$ the surface of the PEC body and $V_{n}$ is the modal excitation coefficient which describes how well the incident field excites each mode. Each $\mathbf{J}_{n}$ is normalized to radiate unit power [9].

The eigenvalue describes the ratio of the reactive power and radiated power of the mode. If the mode is at resonance, the eigenvalue $\lambda_{n}=0$. The eigenvalue can also be expressed as the characteristic angle $a_{n}=180^{\circ}-\tan ^{-1} \lambda_{n}$. If the characteristic angle is over $180^{\circ}$, the mode is storing net electric (capacitive) energy and if it is below $180^{\circ}$, the mode is storing magnetic (inductive) energy in the reactive near field. The mode is at resonance when the characteristic angle is $180^{\circ}$.

The characteristic mode analysis and the full-wave (MoM) simulations in this paper were performed with an in-house MoM solver and the 2018 Altair FEKO software.

\section{B. Quarter-Wave Wavetrap}

In this paper, to illustrate the effectiveness of the theory, decoupling elements called wavetraps were used to increase the isolation between antennas. Wavetraps were previously studied in [17] by using only parametric sweeps.

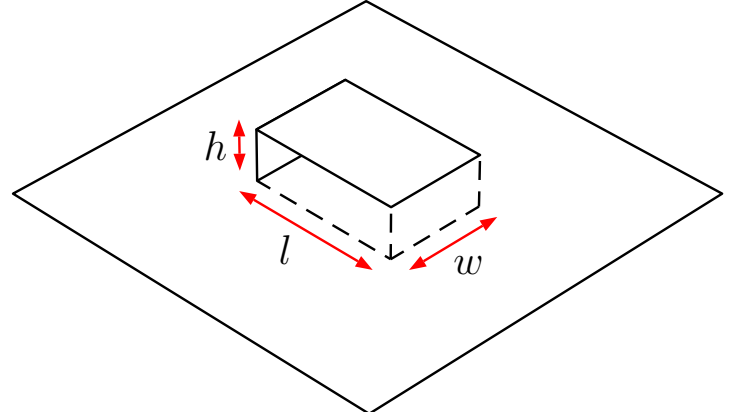

Fig. 1. A planar wavetrap.

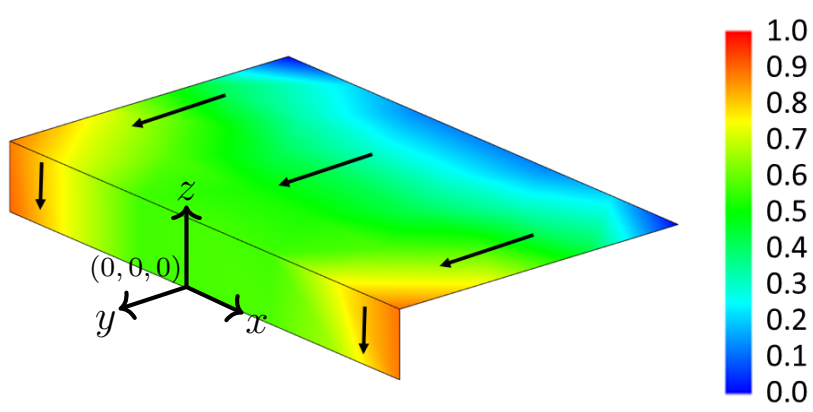

Fig. 2. Surface current of $\mathbf{J}_{1}[\mathrm{~A} / \mathrm{m}]$

A wavetrap is a planar quarter-wavelength patch shortcircuited at one end on a ground plane, as illustrated in Fig. 1. A single wavetrap is defined by its length $l$, height $h$ and width $w$. By utilizing the characteristic mode theory, the fundamental mode of the wavetrap is calculated as $\mathbf{J}_{1}$ and shown in Fig. 2. The eigenvalue and thus the resonance frequency of this mode depend on the height $h$ and the length $l$ of the wavetrap [17]. In Fig. 3, it can be seen that as the length of the wavetrap is swept from $23 \mathrm{~mm}$ to $25 \mathrm{~mm}$, the resonant frequency of the mode is reduced from $2.6 \mathrm{GHz}$ to $2.4 \mathrm{GHz}$. The wavetrap height $h$ is kept at $5 \mathrm{~mm}$. The origin of the coordinate system is in the middle of short-circuited end of the wavetrap as indicated in Fig. 2.

In the example cases of this paper, it is approximated that only the fundamental mode (mode 1) of the wavetrap is excited, and that the current distribution $\mathbf{J}_{1}$ remains fairly consistent when length $l$ of the wavetrap is varied. The validity of these approximations will be discussed in Section II-H.

\section{Scattering of a Decoupling Element}

The electric field of a current distribution on an arbitrary location can be calculated with the equation

$$
\begin{gathered}
\mathbf{E}_{s}=-j \omega \mu \int_{S} G\left(\mathbf{r}, \mathbf{r}^{\prime}\right) \mathbf{J}\left(\mathbf{r}^{\prime}\right) d S^{\prime} \\
-\frac{j}{\epsilon \omega} \nabla \int_{S} G\left(\mathbf{r}, \mathbf{r}^{\prime}\right) \nabla_{s}^{\prime} \mathbf{J}\left(\mathbf{r}^{\prime}\right) d S^{\prime},
\end{gathered}
$$

where $G\left(\mathbf{r}, \mathbf{r}^{\prime}\right)$ is the Green's function, $\omega$ the angular frequency, $\epsilon$ permittivity and $\mu$ permeability. By combining (2) 


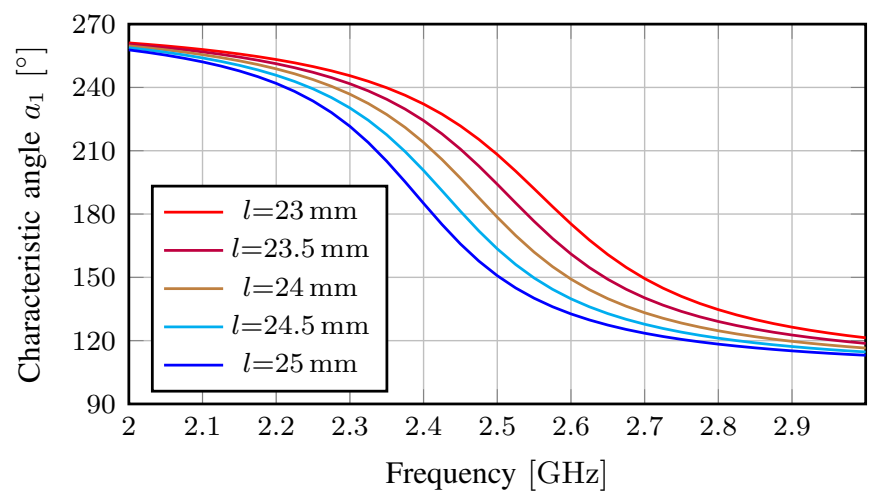

Fig. 3. Characteristic angles of the fundamental mode (mode 1) of a wavetrap with different lengths and the width of $40 \mathrm{~mm}$.

and (3), the following equation for the scattered field of a conducting body is obtained

$$
\mathbf{E}_{s}=\sum_{n} \frac{V_{n}}{1+j \lambda_{n}} \mathbf{E}_{n}
$$

where

$$
\begin{gathered}
\mathbf{E}_{n}=-j \omega \mu \int_{S} G\left(\mathbf{r}, \mathbf{r}^{\prime}\right) \mathbf{J}_{n}\left(\mathbf{r}^{\prime}\right) d S^{\prime} \\
-\frac{j}{\epsilon \omega} \nabla \int_{S} G\left(\mathbf{r}, \mathbf{r}^{\prime}\right) \nabla_{s}^{\prime} \mathbf{J}_{n}\left(\mathbf{r}^{\prime}\right) d S^{\prime},
\end{gathered}
$$

is the modal electric field.

Then, $\lambda_{n}=\tan \left(180^{\circ}-a_{n}\right)$ is substituted in (4) to obtain

$$
\begin{aligned}
\mathbf{E}_{s} & =\sum_{n} \frac{1}{1-j \tan \left(180^{\circ}-a_{n}\right)} V_{n} \mathbf{E}_{n} \\
& =\sum_{n} \sqrt{\frac{1}{1+\tan ^{2} a_{n}}} e^{j\left(a_{n}-180^{\circ}\right)} V_{n} \mathbf{E}_{n} .
\end{aligned}
$$

\section{Farfield Approximation}

If farfield approximation $\left(\left|r-r^{\prime}\right|>>0\right)$ is assumed, the derivative term $\frac{\nabla \nabla}{k^{2}} \rightarrow 0$ and (6) reduces to

$$
\begin{array}{r}
\mathbf{E}_{s}=\sum_{n} \sqrt{\frac{1}{1+\tan ^{2} a_{n}}} e^{j\left(a_{n}-270^{\circ}\right) \frac{\omega \mu e^{-j k r}}{4 \pi r}} \\
V_{n} \mathbf{F}_{n}(\theta, \phi),
\end{array}
$$

where $r=|\mathbf{r}|$ and $\mathbf{F}_{n}(\theta, \phi)$ is the characteristic mode farfield radiation pattern at the angular position of interest. By assuming an incident plane wave on the wavetrap, $\mathbf{E}_{i n c}=$ $E_{0} e^{-j k y} \hat{\mathbf{e}_{z}}$ and that only the fundamental mode (mode 1$)$ is excited in the wavetrap, the cancellation for the $\mathrm{z}$-component of the fields is calculated with

$$
\begin{aligned}
& \frac{E_{t o t, z}(\mathbf{r})}{E_{\text {inc }, z}(\mathbf{r})}=\frac{E_{i n c, z}(\mathbf{r})+E_{s, z}(\mathbf{r})}{E_{i n c, z}(\mathbf{r})} \\
& =1+\frac{\sqrt{\frac{1}{1+\tan ^{2} a_{1}}} e^{j\left(a_{1}-270^{\circ}\right) \frac{\omega \mu e^{-j k r}}{4 \pi r}} V_{1} F_{1, z}(\theta, \phi)}{E_{0} e^{-j k y}}
\end{aligned}
$$

If we consider the cancellation only along the positive $y$ axis, i.e., $\mathbf{r}=(0, y>0,0)$, the fundamental mode (mode 1 ) characteristic farfield is $F_{1}\left(90^{\circ}, 0^{\circ}\right)=1$ and (8) simplifies to

$$
\frac{E_{\text {tot }, z}(y)}{E_{\text {inc, }, z}(y)}=1+\sqrt{\frac{1}{1+\tan ^{2} a_{1}}} e^{j\left(a_{1}-270^{\circ}\right)} \frac{\omega \mu}{4 \pi y} \frac{V_{1}}{E_{0}} .
$$

The term $\sqrt{\frac{1}{1+\tan ^{2} a_{1}}}$ defines the amplitude of the scattered field in terms of the characteristic angle $a_{1}$. When the mode is at resonance, the eigenvalue $\lambda_{1}=0$ and $a_{1}=180^{\circ}$, and the amplitude of scattering reaches its maximum value. When the mode is either inductive or capacitive, i.e., $a_{1}<180^{\circ}$ or $a_{1}>180^{\circ}$ respectively, the amplitude decreases.

In the case of the defined plane wave excitation, $\arg \left(V_{1}\right)=$ 0 . Thus, the term $e^{j\left(a_{n}-270^{\circ}\right)}$ describes the phase of the scattered field with respect to the incident plane wave. When the mode is at resonance, i.e. $a_{n}=180^{\circ}$, the phase difference of the scattered far-field $E_{s, z}$ is $-90^{\circ}$ compared to incident plane wave $E_{\text {inc, } z}$.

When the mode is capacitive, $a_{n}>180^{\circ}$, the phase difference is smaller than $-90^{\circ}$. When the mode is inductive, $a_{n}<180^{\circ}$, the phase difference is larger than $-90^{\circ}$, i.e, the scattered field is destructive to the incident plane wave. Thus, the original field is cancelled by the wavetrap and it is beneficial to design the wavetrap to be inductive.

However, it can be seen from (9) that with lower characteric angles $a_{n}$ the amplitude of the scattered field decreases, as the operating frequency is moved further away from resonance. Thus, there exists an optimal value for $a_{n}$ for cancelling the incident field depending on the distance $r$. However, cancelling fields in the farfield of the wavetrap with plane wave excitation is not very effective as the scattered field of the wavetrap decreases with respect to $1 / r$, whereas the incident field strength remains the same.

\section{E. Modal Near-Field Pattern Effect}

In the previous section, the farfield cancelling scattered field of the wavetrap is considered. However, when studying the cancellation effect near the wavetrap, the phase of the characteristic near-field needs to be taken into account. When calculating the near-field of a wavetrap from the modal currents of the wavetrap using (3), the near-field terms in the integral and the physical size of the current distribution cause extra phase delay in the near-field, when compared to an ideal far-field source for which (7) could be used.

To solve for the exact phase in the near-field, the characteristic near-field is calculated from the characteristic currents. For the planar wavetrap fundamental mode (mode 1 ), the phase of the modal near-field $\arg \left(E_{1, z}\right)$ is presented in Fig. 4.

Using (6), the following equation that shows the effect of the modal near-field phase delay is obtained, here presented for the $z$-component of the fields as

$$
E_{s, z}=\sum_{n} \sqrt{\frac{1}{1+\tan ^{2} a_{n}}} e^{j\left(a_{n}-180^{\circ}+\arg E_{n, z}\right)}\left|E_{n, z}\right| V_{n} .
$$




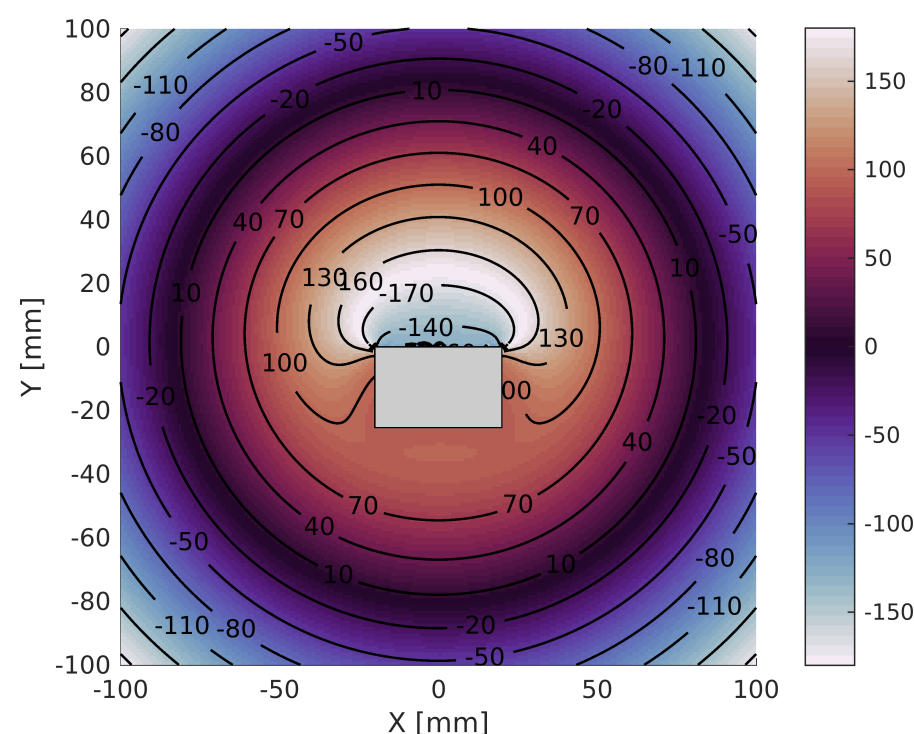

Fig. 4. Phase of the characteristic mode near-field $\arg \left(E_{1, z}\right)$ of the quarterwave wavetrap $(w=40 \mathrm{~mm}, l=25.4 \mathrm{~mm}, z=0 \mathrm{~mm})$.

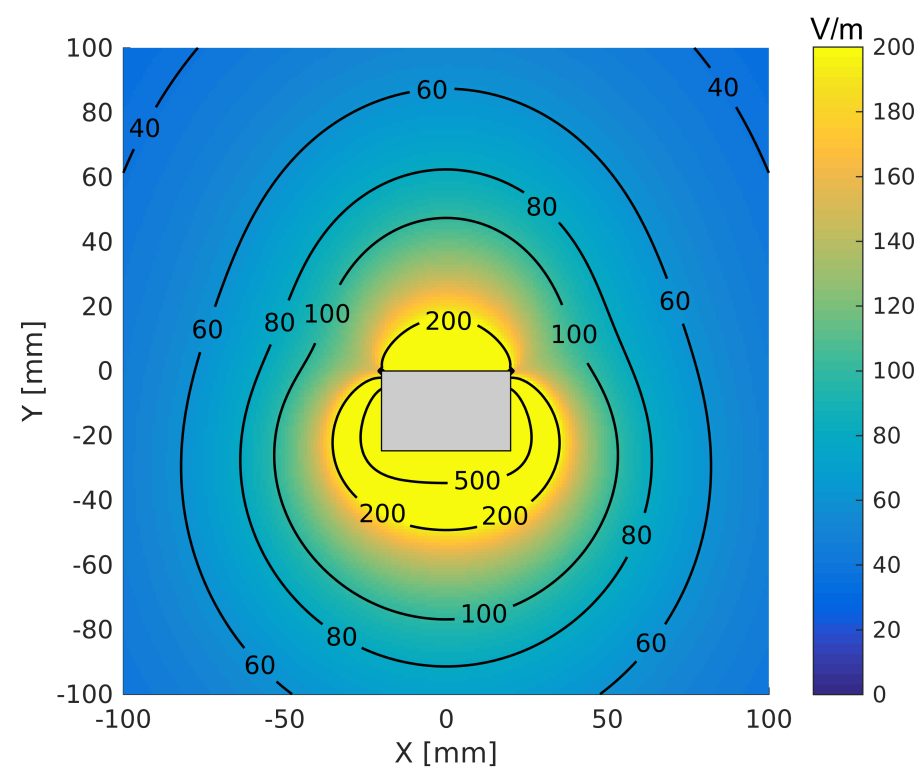

Fig. 5. Magnitude of the characteristic mode near-field $\left|E_{1, z}\right|$ of the quarterwave wavetrap ( $w=40 \mathrm{~mm}, l=25.4 \mathrm{~mm}, z=0 \mathrm{~mm}$ ).

For example, for the planar wavetrap, if the electric field at location $(0,50,0) \mathrm{mm}$ with respect to the wavetrap is targeted for cancellation, the phase and magnitude of the characteristic near-field can be obtained from Figs. 4 and 5 as $E_{1, z}(0,50,0)=95.78 \angle 102.9^{\circ} \mathrm{V} / \mathrm{m}$. With incident plane wave amplitude $E_{0}=1 \mathrm{~V} / \mathrm{m}, V_{1}=0.0037 \angle 0^{\circ}$. Then, the ratio of the total field and the incident field, i.e. the cancellation, is calculated as

$$
\begin{aligned}
& \frac{E_{t o t, z}(\mathbf{r})}{E_{\text {inc }, z}(\mathbf{r})}=\frac{E_{i n c, z}(\mathbf{r})+E_{s, z}(\mathbf{r})}{E_{i n c, z}(\mathbf{r})} \\
& =1+\sqrt{\frac{1}{1+\tan ^{2} a_{1}}} e^{j\left(a_{1}-180^{\circ}+\arg E_{1, z}(\mathbf{r})+k y\right)} V_{1}\left|E_{1, z}\right|
\end{aligned}
$$

160
By plotting the magnitude of this ratio in Fig. 6, it is seen that optimal field cancellation occurs with $a_{1}=135.6^{\circ}$ at $\mathbf{r}=$ $(0,50,0) \mathrm{mm}$. The cancellation is also plotted for distances of $25 \mathrm{~mm}, 10 \mathrm{~mm}$ and $5 \mathrm{~mm}$ from the wavetrap along the $y$-axis with optimal characteristic angles of $135^{\circ}, 134.4^{\circ}$ and $134.3^{\circ}$, respectively. It is seen that better cancellation is obtained close to the wavetrap as the scattered field strength is higher there.

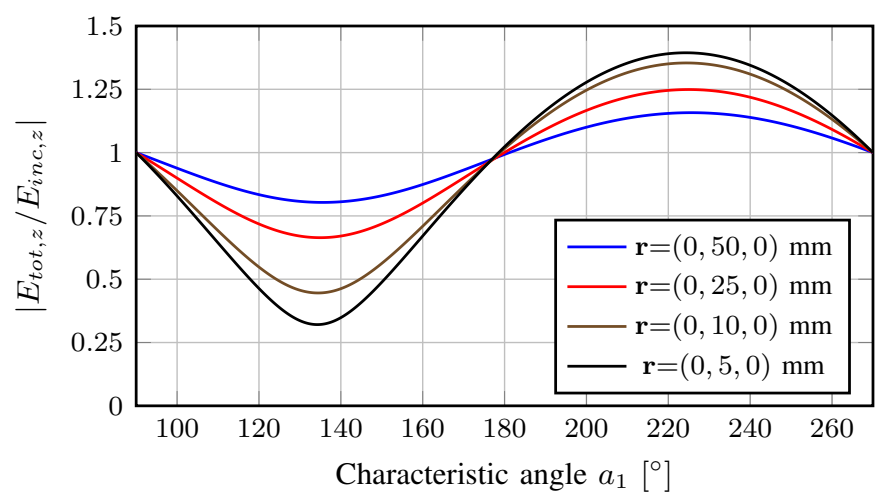

Fig. 6. Cancellation of the near-field $z$-component of the wavetrap at several locations when excited by a plane wave as a function of the characteristic angle $a_{1}$ of a wavetrap ( $w=40 \mathrm{~mm}, l=25.4 \mathrm{~mm}$ ).

\section{F. Modal Excitation Coefficient Effect}

So far, plane wave excitation is considered for the wavetrap. Thus, the phase of the excitation coefficient has always been 00 in phase with the exciting plane wave, i.e, $\arg \left(V_{1}\right)=0^{\circ}$ if $\mathbf{E}_{i n c}=E_{0} e^{-j k y} \hat{\mathbf{e}}_{z}$.

However, if the antenna that is exciting the wavetrap is close to the wavetrap, the exciting wave cannot be approximated with a plane wave, which causes a phase delay in the modal excitation coefficient. This phenomenon can be illustrated by looking at the current distribution $\mathbf{J}_{1}$ of the wavetrap in Fig. 2. The strongest current of this mode occurs along the shorted edges of the wavetrap, to which the distance from the exciting antenna is slightly longer than to the center of the wavetrap, if the phase center of the exciting antenna is located along the $y$-axis. This distance difference causes a phase delay in the excitation of the wavetrap with respect to the wavetrap center.

In addition, if the exciting antenna is close to the region where the incident fields should be cancelled, the exact phase of the radiation of the exciting antenna must be taken into account. This is because the phase of the exciting antenna is non-linear due to the physical aperture size of the antenna and the effect of the near-field terms in the integral equation.

Near the wavetrap, the strength of the scattered field can be higher than the exciting field, especially if the incident field is not a plane wave and its strength is decreasing with distance. Therefore, the exact field strengths of modal near-field $\mathbf{E}_{n}$, incident wave $\mathbf{E}_{i n c}$ and the modal excitation coefficient $V_{n}$ need to be taken into account so that the wavetrap cancels exactly the incident field at the desired location.

By taking these effects into account, the single point field cancellation at a certain location $\mathbf{r}$ with respect to the $z$ component of the original incident field is expressed as 


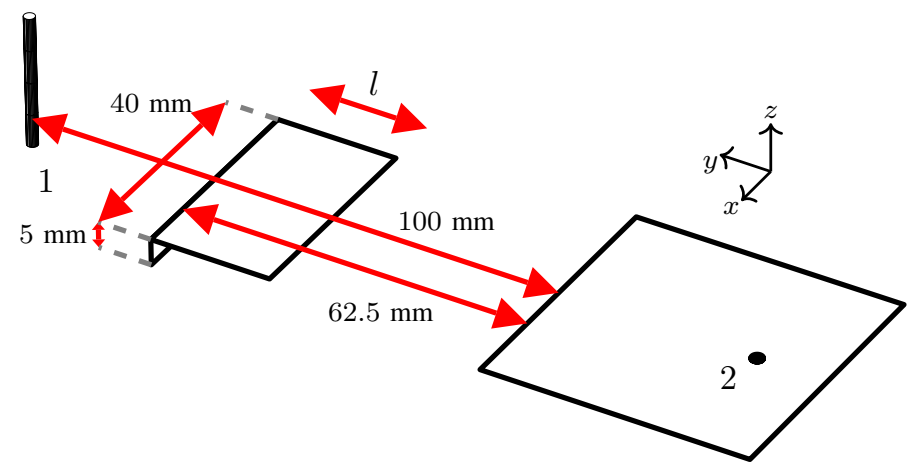

Fig. 7. A patch antenna (antenna 2), monopole antenna (antenna 1) and a wavetrap above an infinite ground plane.

$$
\begin{aligned}
& \frac{E_{t o t, z}(\mathbf{r})}{E_{\text {inc,z }}(\mathbf{r})}=\frac{E_{s, z}(\mathbf{r})+E_{i n c, z}(\mathbf{r})}{E_{i n c, z}(\mathbf{r})}=1+\sum_{n} \sqrt{\frac{1}{1+\tan ^{2} a_{n}}} \\
& e^{i\left(a_{n}-180^{\circ}+\arg E_{n, z}(\mathbf{r})+\arg V_{n}-\arg E_{i n c, z}(\mathbf{r})\right)} \frac{\left|E_{n, z}(\mathbf{r})\right|}{\left|E_{i n c, z}(\mathbf{r})\right|}\left|V_{n}\right|
\end{aligned}
$$

This equation describes the cancellation properties of a wavetrap in the case when the exciting antenna and the desired cancellation point are close to the wavetrap. To illustrate the functionality of the equation, the case illustrated in Fig. 7 is studied. The patch and monopole antennas resonate at 2.6 $\mathrm{GHz}$ and are separated by the distance of $100 \mathrm{~mm}$. The shorted edge of the wavetrap is located at the distance of $62.5 \mathrm{~mm}$ from the patch antenna. These values correspond to antenna separation of $0.87 \lambda_{0}$ and wavetrap distance of $0.54 \lambda_{0}$ from the patch antenna. The field caused by the patch antenna should be cancelled at the location of the monopole to increase the isolation, i.e., the incident field $E_{\text {inc, },}(0,37.5,0)$ caused by antenna 2 should be cancelled. The origin of the coordinate system is at the center of the short-circuited end of the wavetrap as indicated in Fig. 2.

First, only the field of the patch antenna 2 is simulated, and the phase and amplitude of the incident field is obtained as $E_{\text {inc, } z}(0,37.5,0)=2.797 \angle 134.3^{\circ} \mathrm{V} / \mathrm{m}$. Then, the modal excitation coefficient is calculated, i.e., the integral of the incident field with the modal current distribution of the wavetrap, giving $V_{1}=0.0387 \angle-135.37^{\circ}$. From the characteristic nearfield in Figs. 4 and 5, $E_{1, z}(0,37.5,0)=119.5 \angle 139.7^{\circ} \mathrm{V} / \mathrm{m}$ is obtained. By using (12), the cancellation with respect to the characteristic angle is plotted in Fig. 8.

As can be seen, the optimal value for the characteristic angle of the wavetrap is now $a_{1}=128.2^{\circ}$. This corresponds to $l=25.4 \mathrm{~mm}$ for the wavetrap. The optimal characteristic angle is smaller than in Sections II-D and II-E because for values $a>128.2^{\circ}$, the scattered field from the wavetrap is too strong and larger than $E_{i n c, z}$. The simulated S-parameters of the patch and dipole are presented in Fig. 9 with the calculated optimal length of the wavetrap. It is seen that the isolation is improved by $19 \mathrm{~dB}$ at the design frequency of $2.6 \mathrm{GHz}$ by adding the wavetrap with the optimal characteristic angle for the fundamental mode.

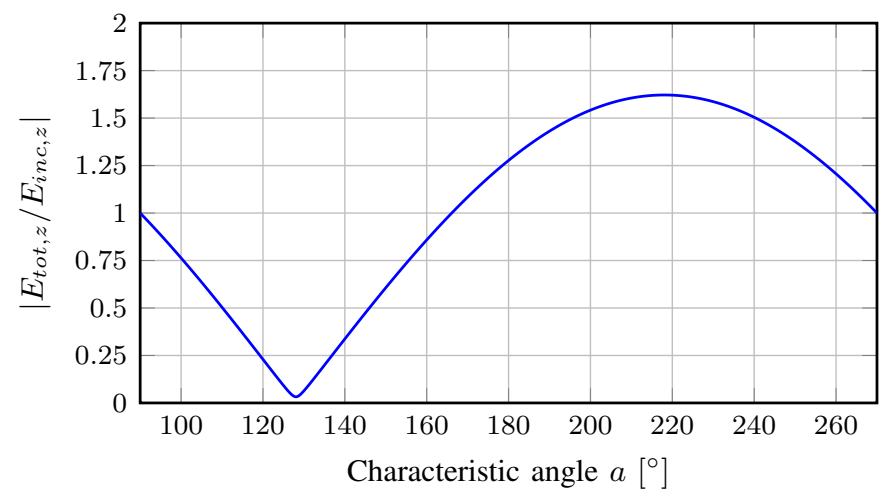

Fig. 8. Cancellation of the near-field $z$-component of the wavetrap at location $(0,37.5,0) \mathrm{mm}$ when excited by a patch antenna as a function of the characteristic angle of a wavetrap.

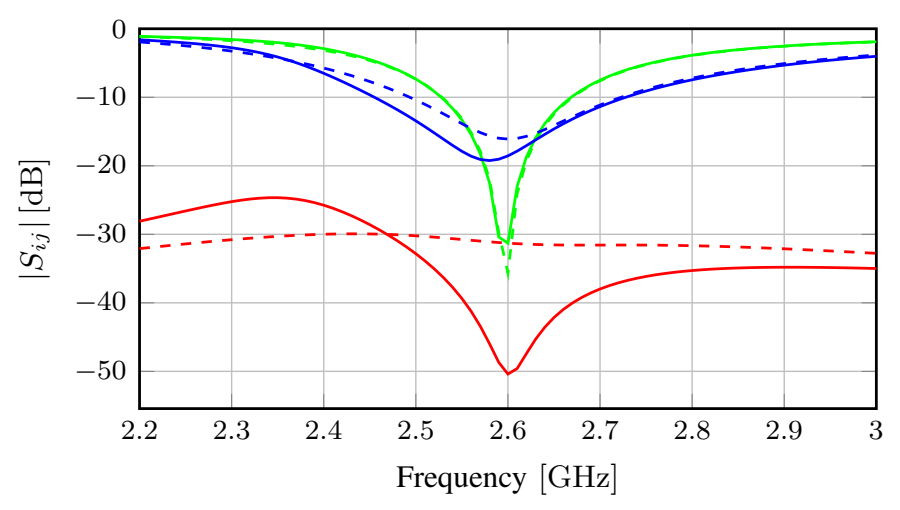

$$
\begin{array}{|l}
- \text { - - } S_{11} \text {, original }-S_{11} \text { with wavetrap } \\
\text { - - - } S_{22} \text {, original }-S_{22} \text { with wavetrap } \\
--S_{21} \text {, original }-S_{21} \text { with wavetrap }
\end{array}
$$

Fig. 9. Simulated S-parameters of a patch antenna and a monopole with and without a wavetrap ( $w=40 \mathrm{~mm}, l=25.4 \mathrm{~mm})$.

\section{G. Multipoint Cancellation}

In cases of more practical antennas, it is not enough to cancel the fields at only one point, as was done for the quarterwave monopole in the previous section. The fields that couple from the transmitting to the receiving antenna need to be cancelled across a larger area around the receiving antenna, i.e., considering the actual current distribution of that antenna. Therefore, the method is extended to optimize the cancellation according to the current distribution on the receiving antenna. As an example of this case, the coupling of two patch antennas on a ground plane is studied. A wavetrap is placed between the patch antennas to increase the isolation (see Fig. 10).

To decouple the patch antennas effectively, the electric fields that excite the current distribution of the receiving patch antenna (antenna 1) need to be cancelled. The magnitude of the total current distribution on the surface of the receiving patch antenna is shown in Fig. 11, when it is excited from the feed port with a $1 \mathrm{~V}$ voltage at $2.6 \mathrm{GHz}$. The patch has mainly $y$-directed currents on the patch and $z$-directed currents on the 


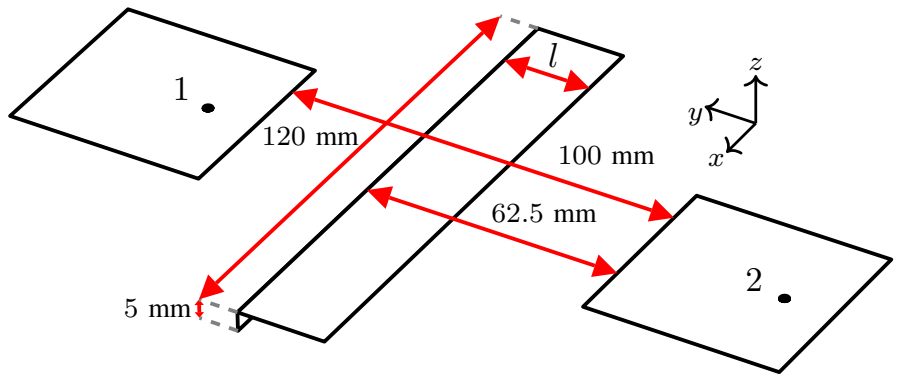

Fig. 10. An infinite ground plane with two patch antennas and a wide wavetrap.

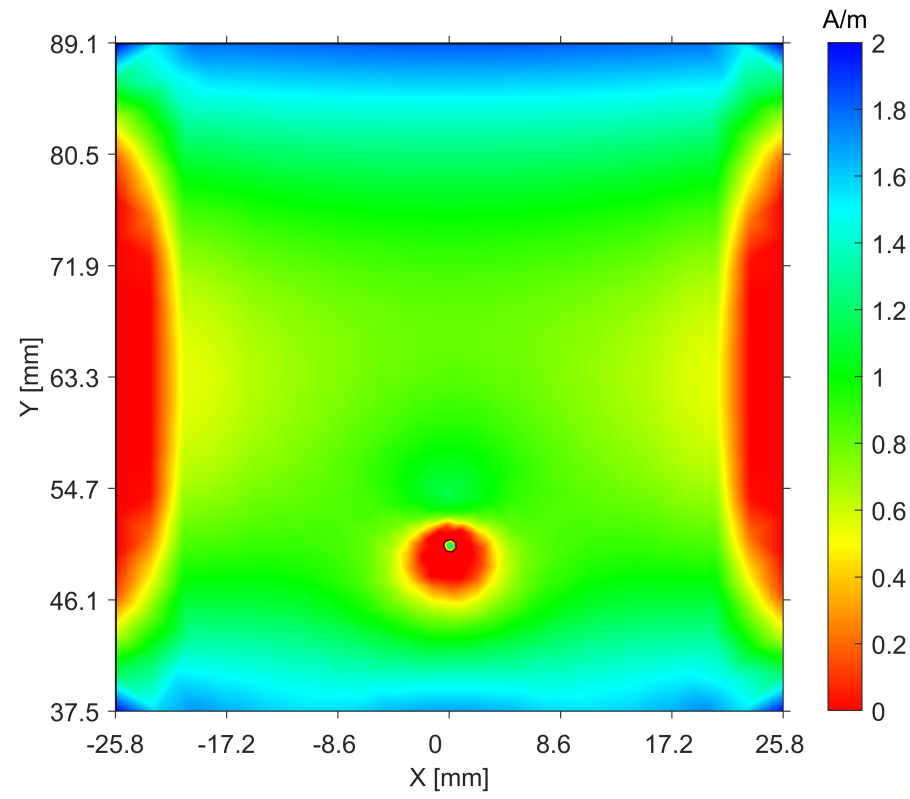

Fig. 11. Surface current $\left\|\mathbf{J}_{p}\right\|$ of the patch antenna 1 with $1 \mathrm{~V}$ feed voltage at $2.6 \mathrm{GHz}$.

feed pin. To decouple the antennas, the fields that excite these currents need to be cancelled. Therefore, the antenna coupling coefficient is calculated with respect to the scattered field from the wavetrap combined with the incident field. The coupling coefficient $M_{t o t}$ is given by

$$
M_{t o t}=\int_{S} \mathbf{J}_{p} \cdot \mathbf{E}_{t o t} d S
$$

Then, the ratio of the coupling with the wavetrap with respect to the original coupling with the incident field can be obtained.

$$
\begin{aligned}
& \frac{M_{t o t}}{M_{i n c}}=\frac{\oiiint_{S} \mathbf{J}_{p} \cdot\left(\mathbf{E}_{i n c}+\mathbf{E}_{s}\right) d S}{\oiiint_{S} \mathbf{J}_{p} \cdot \mathbf{E}_{i n c} d S}=1+\frac{\oiiint_{S} \mathbf{J}_{p} \cdot \mathbf{E}_{s} d S}{\oiiint_{S} \mathbf{J}_{p} \cdot \mathbf{E}_{i n c} d S} \\
& =1+\sum_{n} \sqrt{\frac{1}{1+\tan ^{2} a_{n}}} e^{j\left(a_{n}-180^{\circ}\right)} V_{n} \frac{\oiiint_{S} \mathbf{J}_{p} \cdot \mathbf{E}_{n} d S}{\oiiint_{S} \mathbf{J}_{p} \cdot \mathbf{E}_{i n c} d S}
\end{aligned}
$$

The optimal width of the wavetrap is mainly determined by how well the modal near field matches the original incident

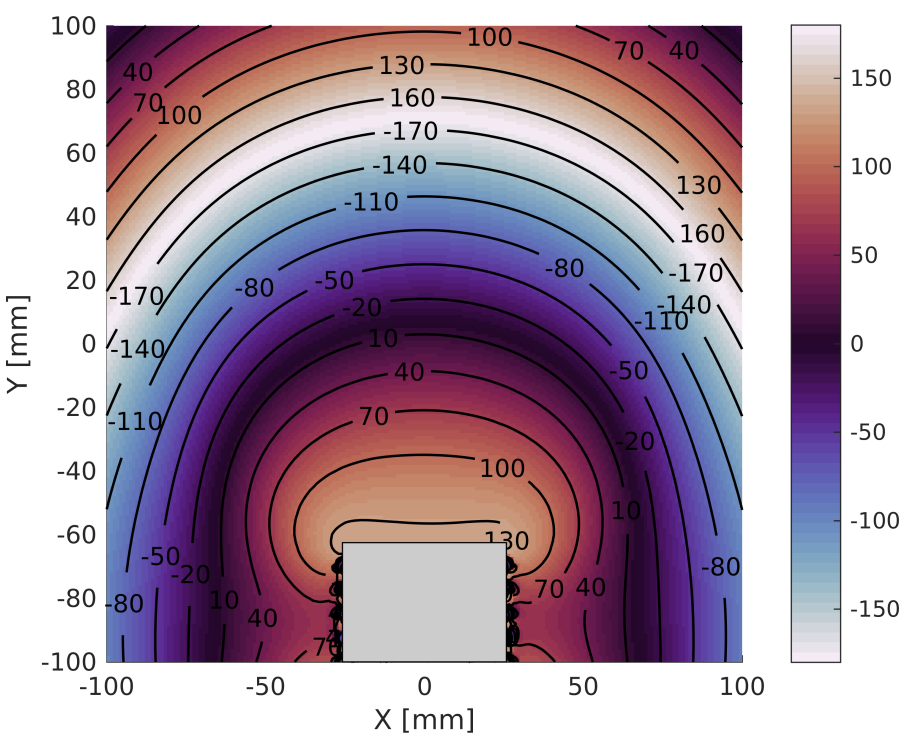

Fig. 12. Phase of the incident field $E_{i n c, y}$ caused by the transmitting patch antenna $2(z=5 \mathrm{~mm})$.

field, i.e., the ratio $\oiiint_{S} \mathbf{J}_{p} \cdot \mathbf{E}_{n} d S / \oiiint_{S} \mathbf{J}_{p} \cdot \mathbf{E}_{\text {inc }} d S$. The modal excitation coefficient $V_{n}$ and the characteristic angle $a_{n}$ have also an effect. The phase of the incident field is plotted in Fig. 12.

The value $\min \left|M_{t o t}\left(a_{1}\right) / M_{i n c}\right|$ is plotted in Fig. 13 for the wavetrap, i.e, the best possible cancellation with any value of $a_{n}$ for each wavetrap width $w$. It is seen there is a minimum at two values of $w$ for the best possible isolation. Out of these minima, the first one at $w=120 \mathrm{~mm}$ is chosen. Figures 14 and 15 show the magnitude and phase of the $y$-component of the fundamental characteristic mode near-field $\mathbf{E}_{1}$ of a 120 $\mathrm{mm}$ wide wavetrap.

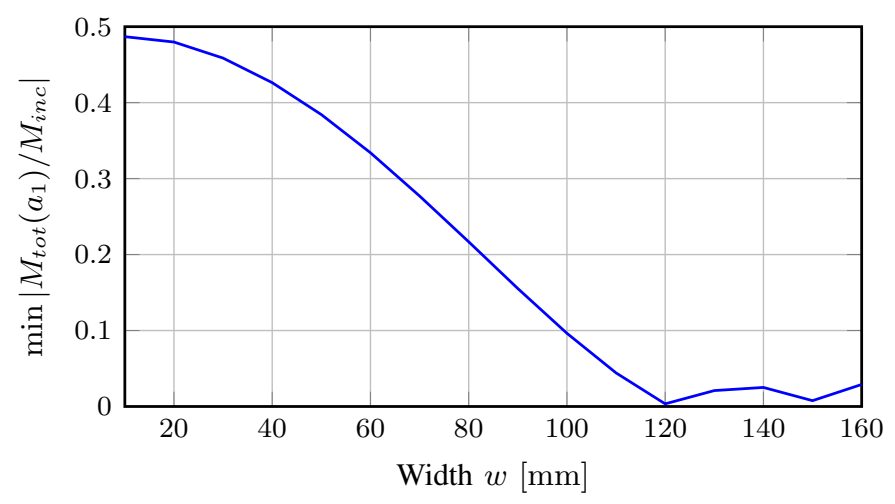

Fig. 13. Best obtainable $\min \left|\frac{M_{t o t}}{M_{\text {inc }}}\right|$ with any value of $a_{1}$ when varying wavetrap width $w$.

The optimal $a_{1}$ for cancellation is then found by plotting (14) in Fig. 16. It is seen that the coupling is well cancelled when $a_{1}=145.5^{\circ}$. This corresponds to $l=25.03 \mathrm{~mm}$ for the wavetrap. S-parameters from the full-wave simulation of the two patch antennas with wavetrap $l=25.03 \mathrm{~mm}$ are shown in Fig. 17. It is seen that the isolation is improved by $33 \mathrm{~dB}$ 


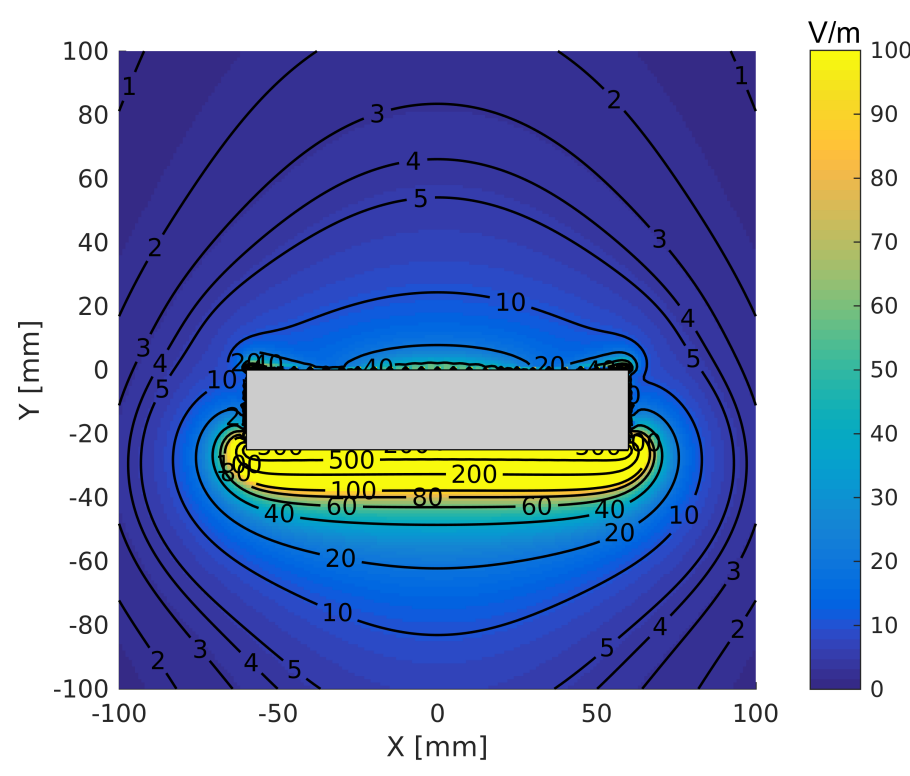

Fig. 14. Magnitude of the characteristic mode near-field $E_{1, y}$ of the quarterwave wavetrap ( $w=120 \mathrm{~mm}, l=25.03 \mathrm{~mm}, z=5 \mathrm{~mm})$.
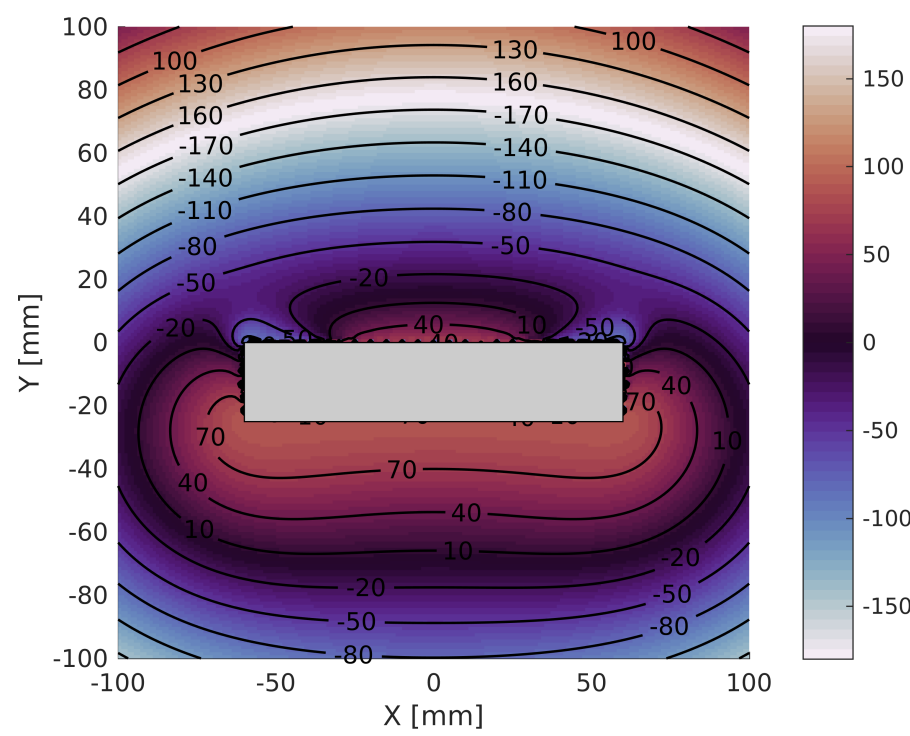

Fig. 15. Phase of the characteristic mode near-field $E_{1, y}$ of the quarter-wave wavetrap ( $w=120 \mathrm{~mm}, l=25.03 \mathrm{~mm}, z=5 \mathrm{~mm}$ ).

at the design frequency of $2.6 \mathrm{GHz}$, and at least $12 \mathrm{~dB}$ within the $142 \mathrm{MHz}-10 \mathrm{~dB}$ bandwidth of the patch antennas.

For comparison, a block of absorber is simulated in place of the wavetrap. The used absorber was ECCOSORB LS22 $\left(\epsilon_{r}=2.2, \tan \delta=1.09\right)$ [18]. With the same dimensions as the wavetrap, only a small isolation improvement of $0.2 \mathrm{~dB}$ was obtained. Thus, the height of the absorber was increased 5 times and the width 2 times to that of the wavetrap resulting in dimensions of $(120 \times 50 \times 25) \mathrm{mm}^{3}$. However, this resulted in only $6 \mathrm{~dB}$ of isolation improvement at the center frequency, as compared to $33 \mathrm{~dB}$ improvement with the wavetrap. This result shows that the proposed method still gives better isolation even though the absorber occupies 10 times the volume of the wavetrap. Moreover, in practice, the larger absorber would increase the overall antenna profile.

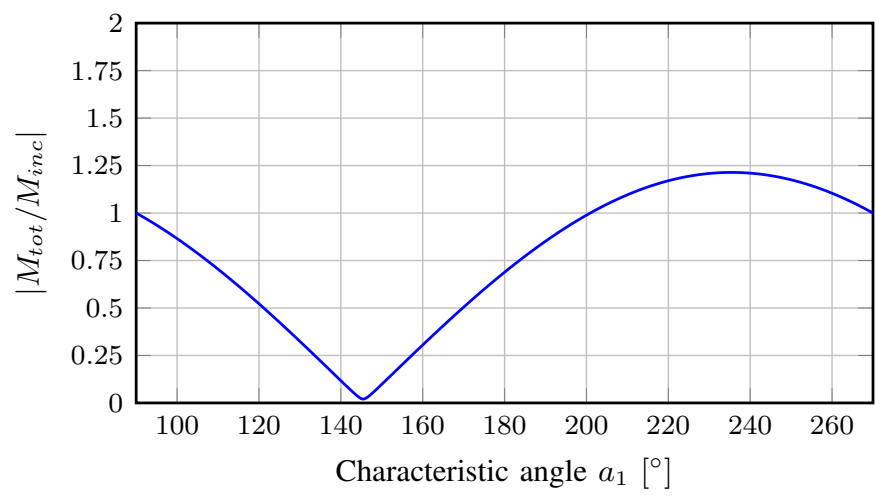

Fig. 16. The coupling of two patch antennas as a function of the characteristic angles $a_{1}$ of a wavetrap $(w=120 \mathrm{~mm})$.

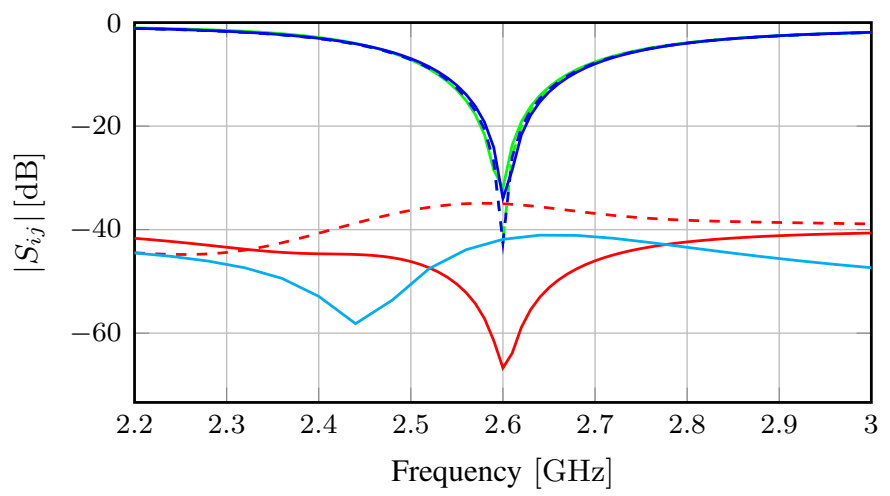

Fig. 17. Simulated S-parameters of two patch antennas with and without a wavetrap $(w=120 \mathrm{~mm}, l=25.03 \mathrm{~mm})$

\section{H. Effect of Used Approximations}

At $2.6 \mathrm{GHz}$, a $z$-polarized incident plane wave mainly excites the fundamental mode (mode 1) of the wavetrap. Including five of the most significant higher order modes changes the phase of the scattered field $E_{1, y}$ by a maximum of $1.6^{\circ}$ at $\mathbf{r}=(0,37.5,0) \mathrm{mm}$ when compared to just using the fundamental mode for the scattering. The change in magnitude of the scattering is below $0.5 \%$ when including the higher order modes. Therefore, in this paper, only the fundamental mode is used in the analysis to ease understanding. For added accuracy, higher order modes can be also included in the analysis, as expressed in the summation in (14).

Another source of error is that changing the wavetrap length $l$ affects not only the characteristic angle $a_{n}$ but also changes slightly the current distribution of the mode $\mathbf{J}_{1}$. This causes a change in the modal excitation coefficient $V_{1}$ and the modal electric field $\mathbf{E}_{1}$ which depend on $\mathbf{J}_{1}$. As the wavetrap length $l$ is varied from 23 to $26 \mathrm{~mm}$, the change in magnitude of $V_{1}$ was below $7.5 \%$ and the change in phase 
below $2.5^{\circ}$, when it is excited with the incident field from the patch antenna. Therefore, for accurate analysis, the search for optimal characteristic angle should be an iterative process, where $a_{n}$ should be evaluated close to the optimal dimensions of the wavetrap.

\section{CONCLUSIONS}

In this paper, a systematic and physically intuitive method for designing antenna decoupling elements, which is based on the theory of characteristic modes, is presented. For validation, a planar wavetrap is designed to improve antenna isolation both in the case of a monopole located close to a patch antenna, and the case of a patch antenna close to a second patch antenna. With the proposed method, the optimal characteristic angle for the wavetrap was predicted, and confirmed with full-wave simulations. With a single-point cancellation model, coupling to the monopole antenna reduced by $19 \mathrm{~dB}$ at its design frequency. With the coupling coefficient model considering field cancellation across a larger area, the isolation between two patch antennas was improved by $33 \mathrm{~dB}$ at their centre frequency and by more than $12 \mathrm{~dB}$ across their 142 $\mathrm{MHz}$ operational bandwidth. As a benchmark, the wavetrap was replaced by an absorber occupying 10 times the volume of the wavetrap. The large structure gives only $6 \mathrm{~dB}$ of isolation improvement, which further validates the effectiveness of the proposed wavetrap method.

So far, decoupling elements such as wavetraps, slots and split-rings, which do not rely on lumped elements, have been designed mainly by parametric sweeps (i.e., brute force). The novel method presented in this paper identifies the effect of characteristic angle, modal electric field and modal excitation coefficient on the obtained antenna isolation, hereby enabling not only an intuitive but also a systematic design and optimization of decoupling elements.

Possible future work includes studying wavetrap arrays which would enable synthesising the cancellation with even more degrees of freedom including also the location of the wavetraps and antennas, which are not systematically optimized in this paper. Multiresonant structures could be studied to extend the bandwidth of the obtained isolation. The analysis in this paper can also be performed also for other types of parasitic resonators to compare their performance in terms of antenna isolation.

\section{REFERENCES}

[1] A. C. Mak, C. R. Rowell, and R. D. Murch, "Isolation enhancement between two closely packed antennas," IEEE Trans. Antennas Propag., vol. 56, no. 11, pp. 3411-3419, Nov. 2008.

[2] M. M. Bait-Suwailam, O. F. Siddiqui, and O. M. Ramahi, "Mutual coupling reduction between microstrip patch antennas using slottedcomplementary split-ring resonators," IEEE Antennas Wireless Propag. Lett., vol. 9, pp. 876-878, 2010.

[3] L. Zhao, L. K. Yeung, and K.-L. Wu, "A coupled resonator decoupling network for two-element compact antenna arrays in mobile terminals," IEEE Trans. Antennas Propag., vol. 62, no. 5, pp. 2767-2776, May 2014.

[4] K. Iwamoto, M. Heino, K. Haneda, and H. Morikawa, "Design of an antenna decoupling structure for an inband full-duplex collinear dipole array," IEEE Trans. Antennas Propag., vol. 66, no. 7, pp. 3763-3768, Jul. 2018.
[5] B. K. Lau and J. B. Andersen, "Simple and efficient decoupling of compact arrays with parasitic scatterers," IEEE Trans. Antennas Propag., vol. 60, no. 2, pp. 464-472, Feb. 2012.

[6] S.-C. Chen, Y.-S. Wang, and S.-J. Chung, "A decoupling technique for increasing the port isolation between two strongly coupled antennas," IEEE Trans. Antennas Propag., vol. 56, no. 12, pp. 3650-3658, Dec, 2008.

[7] S. N. Venkatasubramanian, L. Li, A. Lehtovuori, C. Icheln, and K. Haneda, "Impact of using resistive elements for wideband isolation improvement," IEEE Trans. Antennas Propag., vol. 65, no. 1, pp. 52-62, Jan. 2016

[8] R. Garbacz and R. Turpin, "A generalized expansion for radiated and scattered fields," IEEE Trans. Antennas Propag., vol. 19, no. 3, pp. 348358, May 1971.

[9] R. Harrington and J. Mautz, "Theory of characteristic modes for conducting bodies," IEEE Trans. Antennas Propag., vol. 19, no. 5, pp. 622-628, Sep. 1971.

[10] K. K. Kishor and S. V. Hum, "Multiport multiband chassis-mode antenna design using characteristic modes," IEEE Antennas Wireless Propag. Lett., vol. 16, pp. 609-612, 2016.

[11] H. Li and B. K. Lau, "MIMO systems and antennas for terminals," in Handbook of Antenna Technologies, Z. N. Chen, Ed. Springer, 2015, pp. 1-35.

[12] D. Manteuffel and R. Martens, "Systematic design method of a mobile multiple antenna system using the theory of characteristic modes," IET Microw. Antennas Propag., pp. 887-893, 2014.

[13] P. Liang and Q. Wu, "Characteristic mode analysis of antenna mutual coupling in the near field," IEEE Trans. Antennas Propag., vol. 66, no. 7, pp. 3757-3762, Jul. 2018.

[14] W. Su, Q. Zhang, S. Alkaraki, Y. Zhang, X.-Y. Zhang, and Y. Gao, "Radiation energy and mutual coupling evaluation for multimode MIMO antenna based on the theory of characteristic modes," IEEE Trans. Antennas Propag., vol. 67, no. 1, pp. 74-84, Jan. 2018.

[15] S. Ghosal, A. De, A. Chakrabarty, and R. M. Shubair, "A characteristic mode based decoupling approach," in Proc. IEEE Int. Symp. Antennas Propag. USNC-URSI Radio Sci. Meeting, Atlanta, GA, Jul. 2019.

[16] Z. Miers, "Systematic antenna design using the theory of characteristic modes," Ph.D. dissertation, Lund University, 2016.

[17] M. Heino, S. N. Venkatasubramanian, C. Icheln, and K. Haneda, "Design of wavetraps for isolation improvement in compact in-band full-duplex relay antennas," IEEE Trans. Antennas Propag., vol. 64, no. 3, pp. 10611070, Mar. 2015

[18] E\&C Engineering K.K. Foam sheet high-loss flexible radio wave absorber. [Online]. Available: \{https://ece.co.jp/assets/pdf/products/ ECCOSORB\%20LS_en.pdf $\}$ 